\title{
On Congruences of Galois Representations of Number Fields
}

\author{
by \\ Yoshiyasu OzeKI and Yuichiro TAGUCHI
}

\begin{abstract}
We give a criterion for two $\ell$-adic Galois representations of an algebraic number field to be isomorphic when restricted to a decomposition group, in terms of global representations modulo $\ell$. This is applied to prove a generalization of a conjecture of RasmussenTamagawa [23] under a semistability condition, extending some results [20] of one of the authors. It is also applied to prove a congruence result on the Fourier coefficients of modular forms.
\end{abstract}

2010 Mathematics Subject Classification: Primary 11G35; Secondary 11F80.

Keywords: $\ell$-adic Galois representation, congruence.

\section{$\S 1$. Introduction}

Let $K$ be an algebraic number field, by which we mean a finite extension of $\mathbb{Q}$, and let $G_{K}=\operatorname{Gal}(\bar{K} / K)$ denote its absolute Galois group, where $\bar{K}$ is a fixed algebraic closure of $K$. If $v$ is a finite place of $K$, we choose an extension of $v$ to $\bar{K}$ and denote by $G_{v}$ (resp. $I_{v}$ ) the decomposition (resp. inertia) group of $v$ in $G_{K}$. For an $\ell$-adic representation $V$ of $G_{K}$, where $\ell$ is any prime number, we denote by $V_{v}$ its restriction to $G_{v}$. Motivated by a finiteness conjecture on Abelian varieties of Rasmussen and Tamagawa [23] and related works [20], [24], we prove in this paper the following type of theorems: If the prime $\ell$ is sufficiently large (with respect to $K, v$ and the type of the $\ell$-adic representations under consideration), then for any place $u$ lying above $\ell$ and for any representations $V$ and $V^{\prime}$ satisfying a certain set

Communicated by S. Mochizuki. Received June 5, 2013. Revised September 16, 2013, November 29, 2013, and December 14, 2013.

Y. Ozeki: Research Institute for Mathematical Sciences, Kyoto University,

Kyoto, 606-8502 Japan;

e-mail: yozeki@kurims.kyoto-u.ac.jp

Y. Taguchi: Faculty of Mathematics, Kyushu University,

744, Motooka, Nishi-ku, Fukuoka, 819-0395 Japan;

e-mail: taguchi@math.kyushu-u.ac.jp

(C) 2014 Research Institute for Mathematical Sciences, Kyoto University. All rights reserved. 
of conditions, we have the following implication:

$$
V_{u} \equiv_{\mathrm{ss}} V_{u}^{\prime}(\bmod \ell) \text { and } V_{v} \equiv_{\mathrm{ss}} V_{v}^{\prime}(\bmod \ell) \Rightarrow V_{v} \simeq_{\mathrm{ss}} V_{v}^{\prime} .
$$

Here, we write $V_{v} \equiv_{\mathrm{ss}} V_{v}^{\prime}(\bmod \ell)$ if, for a choice of $G_{v^{-}}$-stable $\mathbb{Z}_{\ell}$-lattices $T$ and $T^{\prime}$ in $V$ and $V^{\prime}$ respectively, $T / \ell T$ and $T^{\prime} / \ell T^{\prime}$ have isomorphic semisimplifications $(T / \ell T)^{\mathrm{ss}}$ and $\left(T^{\prime} / \ell T^{\prime}\right)^{\mathrm{ss}}$ as $\mathbb{F}_{\ell^{-}}$-linear representations of $G_{v}$ (this definition does not depend on the choice of the lattices). We also write $V_{v} \simeq_{\mathrm{ss}} V_{v}^{\prime}$ if their semisimplifications are isomorphic as $\mathbb{Q}_{\ell}$-linear representations of $G_{v}$. See Theorem 3.1 for the precise statement of our main theorem and $\S 3$ for the details of "a certain set of conditions". Roughly speaking, the conditions are:

$-V$ comes from algebraic geometry, and

$-V$ is semistable at $v$.

Furthermore, the "type" of $V$ has to be specified (in particular, bounds are given of the Hodge-Tate weights and of the ramification index needed to acquire semistability at $u$ ). It is important to note that, while imposing an "artificial" bound $b$ on the Hodge-Tate weights of one of the $\left\{V, V^{\prime}\right\}$ (say, $V$ ), we only assume a rather loose and natural bound $(\ell-2) / e^{2}$ of the Hodge-Tate weights of the other one $V^{\prime}$, where $e$ is the absolute ramification index of extensions of $K_{u}$ over which $V$ and $V^{\prime}$ respectively become semistable at $u$.

Our method of proof is, as in [20, §3.3] and [24, Lemma 3.9], to recover the characteristic polynomial of the Frobenius at $v$ from its reduction modulo $\ell$ using the bound of the coefficients, which follows in our case from the condition imposed at $u$ with the help of the theorems of Caruso [5] and Caruso-Savitt [6] (see Thm. 2.6 below). Thus, our proof is obtained by combining certain deep facts in $p$-adic Hodge theory.

If we remove the semistability condition at $v$, we still obtain the coincidence of the set of Weil weights of $V$ and $V^{\prime}$ at $v$; this version, stated as Theorem 3.2, is often enough for applications.

Examples of $V$ as above include the $\mathbb{Q}_{\ell}$-linear dual of the $\ell$-adic étale cohomology group $H_{\mathrm{et}}^{r}\left(X_{\bar{K}}, \mathbb{Q}_{\ell}\right)$ of a smooth proper variety $X$ (in this paper, a variety means a separated scheme of finite type over a field) over $K$ with semistable reduction at $v$ (cf. Prop. 2.8). For such representations, we derive in $\S 4$ several corollaries of Theorem 3.2 in the spirit of the Rasmussen-Tamagawa conjecture. For instance, we prove the following as a special case of Corollary 4.4:

Theorem 1.1. For any integers $b, n \geq 1$, for any prime number $\ell>\left(\begin{array}{c}n \\ {[n / 2]}\end{array}\right) 2^{n b+1}$, for any odd integer $r$ with $1 \leq r \leq b$, and for any smooth proper variety $X$ over $\mathbb{Q}$ which has $r$-th Betti number $\leq n$, has good reduction outside $\ell$ and has semistable reduction at $\ell$, the Galois representation on $H_{\mathrm{et}}^{r}\left(X_{\bar{K}}, \mathbb{Q}_{\ell}\right)$ is not "residually Borel", 
meaning that, for any $G_{\mathbb{Q}^{-}}$stable $\mathbb{Z}_{\ell}$-lattice $T$ in $H_{\mathrm{et}}^{r}\left(X_{\bar{K}}, \mathbb{Q}_{\ell}\right)$, the image of $G_{\mathbb{Q}}$ in $\mathrm{GL}_{\mathbb{F}_{\ell}}(T / \ell T)$ is not contained in a Borel subgroup.

Here, $[n / 2]$ denotes the largest integer not exceeding $n / 2$.

In fact, we also consider $\ell$-adic representations with coefficients. Let $E$ be another algebraic number field, $\lambda$ a finite place of $E$ with residue characteristic $\ell$, and $E_{\lambda}$ the completion of $E$ at $\lambda$. Our main theorems 3.1, 3.2, 3.4 and 3.5 are stated for $E_{\lambda}$-linear representations $V$ and $V^{\prime}$. In Theorems 3.1 and 3.2, where we assume $V_{u} \equiv_{\mathrm{ss}} V_{u}^{\prime}(\bmod \lambda)$ and $V_{v} \equiv_{\mathrm{ss}} V_{v}^{\prime}(\bmod \lambda)$, the constant $C$ giving a lower bound for the primes $\ell$ depends on the coefficient field $E$. If we assume, however, $V_{v} \equiv_{\mathrm{ss}} V_{v}^{\prime}(\bmod \ell)$ rather than $(\bmod \lambda)$, we can eliminate this dependence. This variant, stated as Theorem 3.4, is particularly useful in certain applications. For instance, we can prove the following theorem on the congruence of the Fourier coefficients of modular forms:

Theorem 1.2. Let $k$ be either 1 or an even integer $\geq 2$. Then for any odd squarefree integer $N \geq 1$ and any modular cuspidal normalized Hecke eigenform $f$ on $\Gamma_{0}(N)$ of weight $k$ with Fourier expansion $\sum_{n=1}^{\infty} a_{n} q^{n}$, congruences of the form

$$
a_{p} \equiv p^{i}+p^{j}(\bmod \ell) \quad \text { for all but finitely many primes } p \nmid \ell N
$$

cannot hold if $\ell>2^{2 k}$.

Note that, for each $k$, there are infinitely many $N$ and $f$ as above. See Theorem 5.1 for a more general statement including the case of $\Gamma_{1}(N)$.

In $\S 2$, we recall various notions of weights of $\ell$-adic Galois representations and give some preliminary results needed in later sections. In $\S 3$, we state precisely our main theorems together with the conditions for the Galois representations $V$, and give their proofs. $\S 4$ contains some applications of Theorem 3.2 to étale cohomology groups, and $\S 5$ contains the above mentioned results on the congruence of the Fourier coefficients of modular forms.

\section{$\S 2$. Weights}

We use the same notations $K, E, u, v, \lambda, \ldots$ as in the Introduction. In addition, we use the following notations: $G_{k}$ denotes the absolute Galois group of a field $k$; for a finite place $v$ of $K$, we denote by $K_{v}$ the completion of $K$ at $v$, and identify its absolute Galois group $\operatorname{Gal}\left(\bar{K}_{v} / K_{v}\right)$ with a decomposition group $G_{v}$ of $v ; k_{v}$ denotes the residue field of $v$ and $q_{v}$ its order; $\mathcal{O}_{E}$ and $\mathcal{O}_{E_{\lambda}}$ denote respectively the integer rings of $E$ and $E_{\lambda}$, and $f_{\lambda}$ the absolute residue degree $\left[k_{\lambda}: \mathbb{F}_{\ell}\right]$ of $\lambda ; e\left(K_{u^{\prime}}^{\prime} / K_{u}\right)$ denotes the ramification index of a finite extension $K_{u^{\prime}}^{\prime} / K_{u}$ of discrete valuation fields. 


\section{$\S 2.1$. Weil weights}

Let $v$ be a place of $K$ lying above $\ell$ and let $V$ be a $\mathbb{Q}_{\ell}$-linear representation of $G_{v}$. Choose a lift $\sigma_{v} \in G_{v}$ of the $q_{v}$-th power Frobenius Frob $_{v} \in G_{k_{v}}$ and let $P(T)=\operatorname{det}\left(T-\sigma_{v} \mid V\right)$ be the characteristic polynomial of $\sigma_{v}$ acting on $V$. Recall that an algebraic integer $\alpha$ is said to be a $q$-Weil integer of weight $w$ if $|\iota(\alpha)|=q^{w / 2}$ for any field embedding $\iota: \overline{\mathbb{Q}} \hookrightarrow \mathbb{C}$, where $|\cdot|$ denotes the absolute value of $\mathbb{C}$.

Definition 2.1. We say that $V$ is of type (W) at $v$ if all the roots of $P(T)$ are $q_{v}$-Weil integers. If this is the case, we call the weights of the roots of $P(T)$ the Weil weights of $V$ at $v$, and denote by $\mathrm{W}_{v}(V)$ the multi-set consisting of them.

This definition does not depend on the choice of the Frobenius lift $\sigma_{v}$. Also, the multi-set $\mathrm{W}_{v}(V)$ is unchanged under a finite extension of the base field $K_{v}$.

For example, if $X$ is a proper smooth variety over $K_{v}$, then the $\mathbb{Q}_{\ell}$-linear dual $V=H_{\mathrm{et}}^{r}\left(X_{\bar{K}_{v}}, \mathbb{Q}_{\ell}\right)^{*}$ of the $r$-th $\ell$-adic étale cohomology group of $X_{\bar{K}_{v}}:=X \otimes_{K_{v}} \bar{K}_{v}$ is of type (W). This is known from [9] if $X$ has good reduction at $v$, from [22] and [25] if $X$ has semistable reduction at $v$, and the general case is reduced to the semistable case by de Jong's alteration [7] (cf. [1, proof of Prop. 6.3.2] or [20, proof of Prop. 3.3]).

Now suppose $V$ is an $E_{\lambda}$-linear representation of $G_{v}$. We say that the representation $V$ is semistable at $v$ if the inertia subgroup $I_{v}$ acts unipotently on $V$ (or equivalently, trivially on the semisimplification $V^{\text {ss }}$ of $V$ as an $E_{\lambda}\left[G_{v}\right]$-module; here, we include the case where the action of $I_{v}$ on $V$ is trivial). In general, any $V$ is potentially semistable in the sense that the action of $I_{v}$ on $V$ is quasi-unipotent ([33, Appendix]); thus there exists a finite extension $K_{v^{\prime}}^{\prime} / K_{v}$ such that the restriction of $V$ to $G_{K_{v^{\prime}}}$ is semistable. Hence we can consider the characteristic polynomial $P^{\prime}(T)=\operatorname{det}\left(T-\right.$ Frob $\left._{v^{\prime}} \mid V^{\text {ss }}\right)$ of the Frobenius Frob $v_{v^{\prime}}$ at $v^{\prime}$ acting on the $E_{\lambda}$-vector space $V^{\text {ss }}$. Note that the characteristic polynomial taken with $V^{\text {ss }}$

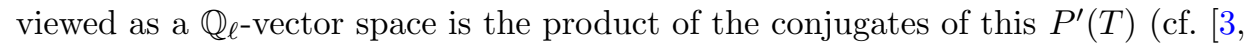
Chap. $8, \S 12$, Prop. 7$]$; here, a conjugate of a polynomial in $E_{\lambda}[T]$ means the result of the coefficientwise application of a $\sigma \in \operatorname{Hom}_{\mathbb{Q}_{\ell}}\left(E_{\lambda}, \overline{\mathbb{Q}}_{\ell}\right)$.)

Definition 2.2. An $E_{\lambda}$-linear representation $V$ of $G_{v}$ is said to be $E$-integral at $v$ if, for any finite extension $K_{v^{\prime}}^{\prime} / K_{v}$ for which the inertia action on $V$ is unipotent, the characteristic polynomial $P^{\prime}(T)$ defined as above has coefficients in $\mathcal{O}_{E}$.

Note that an $E$-integral representation of type (W) at $v$ has Weil weights $\geq 0$ at $v$.

For example, if $X$ is a proper smooth variety over $K_{v}$, then the $\mathbb{Q}_{\ell}$-linear dual $V=H_{\mathrm{et}}^{r}\left(X_{\bar{K}_{v}}, \mathbb{Q}_{\ell}\right)^{*}$ of the $r$-th $\ell$-adic étale cohomology group of $X_{\bar{K}_{v}}:=X \otimes_{K_{v}} \bar{K}_{v}$ 
is conjectured to be $\mathbb{Q}$-integral (cf. $\left[29, \mathrm{C}_{4}\right]$ ). This conjecture is known to be true under the assumption of the existence of the Künneth projector ([25, Cor. 0.6(1)]).

We note here that, by the next lemma, there are totally ramified extensions among the finite extensions $K_{v^{\prime}}^{\prime} / K_{v}$ as above (so that, when we want to compare the characteristic polynomials $P^{\prime}(T)$ for different $V^{\prime}$ s, we can use a $K_{v^{\prime}}^{\prime}$ with residue degree 1$)$ :

Lemma 2.3. If $L / K_{v}$ is a finite Galois extension, then there exists a totally ramified subextension $L^{\prime} / K_{v}$ of $L / K_{v}$ such that $L=L^{\prime} L_{0}$, where $L_{0}$ is the maximal unramified subextension of $L / K_{v}$.

Proof. If $L / K_{v}$ is abelian, this is a consequence of local class field theory (e.g. [17, Th. 7.1]). Suppose $L / K_{v}$ is non-abelian. Let $\sigma$ be a lift in $\operatorname{Gal}\left(L / K_{v}\right)$ of the Frobenius in $\operatorname{Gal}\left(L_{0} / K_{v}\right)$, and set $H:=\langle\sigma\rangle$. Then we have $H \subsetneq \operatorname{Gal}\left(L_{0} / K_{v}\right)$, and the extension $L^{H} / K_{v}$ is a non-trivial totally ramified subextension of $L / K_{v}$. Repeating this process with $L / K_{v}$ replaced by $L / L^{H}$, we are reduced to the case of abelian $L / K_{v}$.

This lemma will be used in the proofs of Lemmas 3.6, 3.7 and the main theorems.

\section{$\S 2.2$. Hodge-Tate weights}

Recall that $u$ is a finite place of $K$ lying above $\ell$. A $\mathbb{Q}_{\ell}$-linear representation $V$ of $G_{u}$ is said (cf. $[12, \S 3.7]$ ) to be of Hodge-Tate type of Hodge-Tate weights $h_{1}, \ldots, h_{n}$, where $n=\operatorname{dim}_{\mathbb{Q}_{\ell}}(V)$ and $h_{i}$ are integers, if $V \otimes_{\mathbb{Q}_{\ell}} \mathbb{C}_{\ell} \simeq \mathbb{C}_{\ell}\left(h_{1}\right) \oplus \cdots \oplus \mathbb{C}_{\ell}\left(h_{n}\right)$ as $\mathbb{C}_{\ell \text {-semilinear }} G_{u}$-representations, where $\mathbb{C}_{\ell}(h)$ denotes the $h$-th Tate twist of the completion $\mathbb{C}_{\ell}$ of a fixed algebraic closure $\overline{\mathbb{Q}}_{\ell}$ of $\mathbb{Q}_{\ell}$. If this is the case, let $\operatorname{HT}_{u}(V)$ denote the multi-set of Hodge-Tate weights of $V$. Note that, by definition (or the well-definedness) of Hodge-Tate weights, $\mathrm{HT}_{u}(V)$ is unchanged under a finite extension of the base field $K_{u}$.

\section{$\S 2.3$. Tame inertia weights}

Let $I_{u}^{\text {tame }}$ denote the tame inertia group of $K$ at $u$ (= the quotient of the inertia group $I_{u}$ at $u$ by its maximal pro- $\ell$ subgroup). A character $\varphi: I_{u}^{\text {tame }} \rightarrow \mathbb{F}_{\ell^{h}}^{\times}$can be written in the form $\varphi=\psi_{1}^{t_{1}} \cdots \psi_{h}^{t_{h}}$, where $\psi_{i}$ are the fundamental characters of level $h([30, \S 1.7])$ and $0 \leq t_{i} \leq \ell-1$. Then we set $\operatorname{TI}_{u}(\varphi):=\left\{t_{1} / e, \ldots, t_{h} / e\right\}$ (as a multi-set), where $e=e\left(K_{u} / \mathbb{Q}_{\ell}\right)$ is the ramification index of $K / \mathbb{Q}$ at $u$. Note that, by $[30, \S 1.4], \mathrm{TI}_{u}(\varphi)$ is unchanged by a "moderately" ramified extension of $K_{u}$; precisely speaking, if $K_{u^{\prime}}^{\prime} / K_{u}$ is a finite extension of ramification index $e\left(K_{u^{\prime}}^{\prime} / K_{u}\right)<(\ell-1) / \max \left\{t_{j} \mid 1 \leq j \leq h\right\}$, then $\operatorname{TI}_{u^{\prime}}\left(\left.\varphi\right|_{I_{u^{\prime}}^{\text {tame }}}\right)=\operatorname{TI}_{u}(\varphi)$. 
Let $V$ be a $\mathbb{Q}_{\ell}$-linear representation of $G_{u}$, and $T$ a $G_{u}$-stable $\mathbb{Z}_{\ell}$-lattice of $V$. Set $\bar{T}:=T / \ell T$. Then its semisimplification $\bar{T}^{\text {ss }}$ (as an $\mathbb{F}_{\ell}\left[G_{u}\right]$-module) is tamely ramified (note that its isomorphism class does not depend on the choice of $T$; cf. $\left[32, \S 15.2\right.$, Thm. 32]) and the action of the tame inertia group $I_{v}^{\text {tame }}$ is described by a sum of characters $\varphi_{i}: I_{v}^{\text {tame }} \rightarrow \mathbb{F}_{\ell^{h_{i}}}^{\times}$. Then we define $\mathrm{TI}_{u}(V)$ (as a multi-set) to be the union of the $\operatorname{TI}_{u}\left(\varphi_{i}\right)$ for all $i$.

\section{$\S 2.4$. Weights of geometric Galois representations}

Let $V$ be a $\mathbb{Q}_{\ell}$-linear representation of $G_{K}$. For any multi-set $X$, we write

$$
\Sigma(X):=\sum_{x \in X} x
$$

whenever the sum on the right-hand side has a meaning.

Definition 2.4. We say that $V$ is of type $(\mathrm{G})$ if it is of type $(\mathrm{W})$ at $v$, of HodgeTate type at $u$, and

$$
\Sigma\left(\mathrm{W}_{v}(V)\right)=2 \Sigma\left(\mathrm{HT}_{u}(V)\right)
$$

If this is the case, we denote this value by $w(V)$ and call it the total weight of $V$.

Note that $\Sigma\left(\mathrm{W}_{v}(V)\right)$ and $\Sigma\left(\mathrm{HT}_{u}(V)\right)$ are respectively the Weil and HodgeTate weights of $\operatorname{det}_{\mathbb{Q}_{\ell}}(V)$.

Typical examples of $V$ of type $(\mathrm{G})$ include the Tate twists $\mathbb{Q}_{\ell}(r)$ for $r \in \mathbb{Z}$ and their twists by characters of finite order; their total weights are $2 r$.

A priori, the notion of type $(\mathrm{G})$ depends on the places $v \nmid \ell$ and $u \mid \ell$ (so it should be called, say, type $\left.\left(\mathrm{G}_{u, v}\right)\right)$, but in practice (i.e., in case $V$ comes from algebraic geometry), it is independent of the places, as the following proposition shows. Its proof, which is modeled on the proof of Lemma 2.1 of [26], has been communicated to us by Yoichi Mieda, to whom we are grateful:

Proposition 2.5. Let $X$ be a proper smooth variety over $K$. Let $V=$ $H_{\mathrm{et}}^{r}\left(X_{\bar{K}}, \mathbb{Q}_{\ell}\right)^{*}$ be the $\mathbb{Q}_{\ell}$-linear dual of the $r$-th $\ell$-adic étale cohomology group of $X_{\bar{K}}:=X \otimes_{K} \bar{K}$, and put $n=\operatorname{dim}_{\mathbb{Q}_{\ell}}(V)$. Then:

(i) $\operatorname{det}(V)$ is isomorphic to the twist of $\mathbb{Q}_{\ell}(n r / 2)$ by a character $\varepsilon$ of order at most 2. If $r$ is odd, then $\varepsilon=1$.

(ii) $V$ is of type $(\mathrm{G})$ with respect to any finite places $u \mid \ell$ and $v \nmid \ell$ of $K$.

Note that, in (i), the Betti number $n$ is even if $r$ is odd by, say, Hodge symmetry. 
Proof. (ii) follows from (i) and the fact that $V$ is of type (W) (here, the fact that $V$ is Hodge-Tate at every place $v \mid \ell$ follows from [11], [35], etc.). To show (i), consider the character $\varepsilon: G_{K} \rightarrow \mathbb{Q}_{\ell}^{\times}$defined by $\operatorname{det}(V)(-n r / 2)$, where $(-n r / 2)$ denotes the $(-n r / 2)$-th Tate twist. If $v \nmid \ell$ is a finite place of $K$ where $X$ has good reduction, then by [9], $V$ is $\mathbb{Q}$-integral and has all Weil weights equal to $r$. Hence $\varepsilon\left(\right.$ Frob $\left._{v}\right)$ is a Weil integer in $\mathbb{Q}$ of weight 0, i.e., a unit of $\mathbb{Z}$. Since Frob ${ }_{v}$ 's for such $v$ 's are dense in $G_{K}$, we see that $\varepsilon$ takes values in $\mathbb{Z}^{\times}$. The second statement of (i) follows from Corollary 3.3.5 of [34].

As the next theorem shows, we can expect in some cases the total weight $w(V)$ to be equal also to $2 \Sigma\left(\mathrm{TI}_{u}(V)\right)$. We refer the reader to, e.g., [12, §5.1.4] for the definition of semistability of an $\ell$-adic representation of $G_{u}$. Note that "semistable" implies "Hodge-Tate". Note also that, if a smooth proper variety $X$ over $K_{u}$ has semistable reduction, then the $\ell$-adic cohomology group $H_{\mathrm{et}}^{r}\left(X_{\bar{K}}, \mathbb{Q}_{\ell}\right)$ and its $\mathbb{Q}_{\ell^{-}}$ linear dual are semistable in this sense; this is a consequence of Fontaine-Jannsen's conjecture $\left(\mathrm{C}_{\mathrm{st}}\right)$ ([12, Conj. 6.2.7]) proved by Tsuji [35].

Theorem 2.6. Let $V$ be a $\mathbb{Q}_{\ell}$-linear semistable representation of $G_{u}$ with $\operatorname{HT}_{u}(V)$ $\subset[0, b]$. If $e\left(K_{u} / \mathbb{Q}_{\ell}\right) b<\ell-1$, then:

(i) $\left(\left[5\right.\right.$, Thms. 1.0.3 and 1.0.5]) $\mathrm{TI}_{u}(V) \subset[0, b]$.

(ii) $\left(\left[6, \mathrm{Thm}^{1}\right]\right) \Sigma\left(\mathrm{HT}_{u}(V)\right)=\Sigma\left(\mathrm{TI}_{u}(V)\right)$.

If $V$ is not assumed to come from algebraic geometry, the equality ( $G$ ) holds in some special cases:

Lemma 2.7. Let $v$ be a finite place of $K$ not lying above $\ell$. If $V$ is a $\mathbb{Q}_{\ell}$-linear representation of $G_{K}$ which is of type $(\mathrm{W})$ at $v$, of Hodge-Tate type at all $u \mid \ell$, and such that the value of $\Sigma\left(\mathrm{HT}_{u}(V)\right)$ is the same for all $u \mid \ell$, then $V$ is of type $(\mathrm{G})$.

Note that the assumption on the Hodge-Tate type is automatic if there is only one place of $K$ lying above $\ell$. Note also that $V=H_{\mathrm{et}}^{r}\left(X_{\bar{K}}, \mathbb{Q}_{\ell}\right)^{*}$ satisfies the condition of this lemma (this follows from the theory of Hodge-Tate decomposition).

Proof. By taking the determinant, we are reduced to the case $\operatorname{dim}_{\mathbb{Q}_{\ell}}(V)=1$. By [28, Chap. III, $\S 2.2$, Cor.], $V$ is unramified outside a finite set $S$ of places. If $V$ has Hodge-Tate weights $r$ at every $u \mid \ell$, then $V(-r)$ has Hodge-Tate weights 0 , and by $[27, \S 5$, Cor. 1], the image of inertia at $u \mid \ell$ is finite. By [16, $\S 2$, Thm. 1], the image of inertia at any place outside $\ell$ is also finite. Thus $V(-r)$ gives rise to an abelian extension of $K$ which is unramified outside $S$ and finitely ramified at all 
places. By class field theory, such an extension is finite. Hence $V$ is isomorphic to a twist by a finite character of $\mathbb{Q}_{\ell}(r)$. Thus $(\mathrm{G})$ holds for $V$.

If there are more than one place above $\ell$, the equality $(\mathrm{G})$ may not hold even for representations coming from algebraic geometry. For example, let $K$ be an imaginary quadratic field, $E$ an elliptic curve over $K$ such that $\operatorname{End}_{K}(E) \otimes_{\mathbb{Z}} \mathbb{Q} \simeq K$, and $\ell$ a prime number which splits in $K$ as $\ell=\lambda \lambda^{\prime}$. Let $V$ be a one-dimensional $G_{K}$-subrepresentation of the $\ell$-adic Tate module $T_{\ell}(E) \otimes_{\mathbb{Z}_{\ell}} \mathbb{Q}_{\ell}$ of $E$. Then $V$ is of type $(\mathrm{W})$ of Weil weight 1 at any $v \nmid \ell$, while it is of Hodge-Tate type of Hodge-Tate weight 0 or 1 at $\lambda$.

If we do not assume the equality $(\mathrm{G})$, we can in fact prove an equality which is fairly close to $(\mathrm{G})$ under a mild condition:

Proposition 2.8. Let $V$ be a $\mathbb{Q}_{\ell}$-linear representation of $G_{K}$ and $q$ a prime number $\neq \ell$. Assume $V$ is of type $(\mathrm{W})$ at all places $v \mid q$ and of Hodge-Tate type at all places $u \mid \ell$. Then

$$
\sum_{v \mid q}\left[K_{v}: \mathbb{Q}_{q}\right] \Sigma\left(\mathrm{W}_{v}(V)\right)=2 \sum_{u \mid \ell}\left[K_{u}: \mathbb{Q}_{\ell}\right] \Sigma\left(\mathrm{HT}_{u}(V)\right) .
$$

Proof. The induced representation $\operatorname{Ind}_{G_{K}}^{G_{\mathbb{Q}}}(V)$ is a representation of $G_{\mathbb{Q}}$ which is of type (W) at $q$ and of Hodge-Tate type at $\ell$, and hence we have

$$
\Sigma\left(\mathrm{W}_{q}\left(\operatorname{Ind}_{G_{K}}^{G_{\mathbb{Q}}}(V)\right)\right)=2 \Sigma\left(\mathrm{HT}_{\ell}\left(\operatorname{Ind}_{G_{K}}^{G_{\mathbb{Q}}}(V)\right)\right)
$$

by Lemma 2.7. We then observe that

$$
\begin{aligned}
\mathrm{W}_{q}\left(\operatorname{Ind}_{G_{K}}^{G_{\mathbb{Q}}}(V)\right) & =\coprod_{v \mid q}\left[K_{v}: \mathbb{Q}_{q}\right] \mathrm{W}_{v}(V), \\
\mathrm{HT}_{\ell}\left(\operatorname{Ind}_{G_{K}}^{G_{\mathbb{Q}}}(V)\right) & =\coprod_{u \mid \ell}\left[K_{u}: \mathbb{Q}_{\ell}\right] \mathrm{HT}_{\ell}(V),
\end{aligned}
$$

where the multiple $m X$ of a multi-set $X$ by a positive integer $m$ is defined in the obvious manner. Indeed, we have

$$
\left.\left(\operatorname{Ind}_{G_{K}}^{G_{\mathbb{Q}}}(V)\right)\right|_{G_{q}}=\bigoplus_{v \mid q} \operatorname{Ind}_{G_{v}}^{G_{q}}\left(\left.V\right|_{G_{v}}\right)
$$

by Mackey's formula ([32, $\S 7.3$, Prop. 22]), and

$$
\mathrm{W}_{q}\left(\operatorname{Ind}_{G_{v}}^{G_{q}}\left(\left.V\right|_{G_{v}}\right)\right)=\left[K_{v}: \mathbb{Q}_{q}\right] \mathrm{W}_{v}\left(\left.V\right|_{G_{v}}\right)
$$

by definition of the induced representation and by the invariance of the Weil weights under finite extensions of the base field. Similar equalities hold for $u \mid \ell$ and $\operatorname{Ind}_{G_{u}}^{G_{\ell}}\left(\left.V\right|_{G_{u}}\right)$. 


\section{§3. Main results}

Let the notations $K, E, u, v, \lambda, \ldots$ be the same as before. In addition, let $n$ and $e$ be fixed integers $\geq 1$ and $b \geq 0$ be a fixed real number. All $E_{\lambda}$-linear representations $V$ in this section are assumed to have dimension $n$. Assume also that $e$ is divisible by the absolute ramification index $e\left(K_{u} / \mathbb{Q}_{\ell}\right)$ of $K_{u} / \mathbb{Q}_{\ell}$.

For the first version of our main theorem, we shall consider $E_{\lambda}$-linear representations $V$ of $G_{K}$ which satisfy the following:

\section{Conditions (I).}

$\left(\mathrm{SS}_{v}\right) V$ is semistable at $v$ (in the sense that the action of the inertia is unipotent; cf. $\S 2.1$,

$\left(\operatorname{Int}_{v}\right) V$ is E-integral at $v$ in the sense of Definition 2.2,

$\left(\mathrm{PST}_{u}\right) V$ becomes semistable (in the sense of Fontaine [12, §5.1.4]) over a finite extension $K_{u^{\prime}}^{\prime}$ of $K_{u}$ whose absolute ramification index $e\left(K_{u^{\prime}}^{\prime} / \mathbb{Q}_{\ell}\right)$ divides $e$ (hence, in particular, $V$ is of Hodge-Tate type at $u$ ),

$\left(\mathrm{HT}_{u}\right) V$ has Hodge-Tate weights $\operatorname{HT}_{u}(V) \subset\left[0,(\ell-1) / e^{2}\right]$, and

$\left(\mathrm{G}_{u, v}\right) V$ is of type $(\mathrm{G})$ in the sense of Definition 2.4.

Our first main result is:

Theorem 3.1. For any $K, E, n, b, e, v$ as above, there exists a constant $C=$ $C\left([E: \mathbb{Q}], n, b, e, q_{v}\right)$ such that the following holds: For any prime number $\ell>C$, any places $u$ of $K$ and $\lambda$ of $E$ both lying above $\ell$, and any representations $V$ and $V^{\prime}$ satisfying Conditions (I) with $\operatorname{HT}_{u}(V) \subset[0, b]$, if one has $V_{u} \equiv_{\mathrm{ss}} V_{u}^{\prime}(\bmod \lambda)$ and $V_{v} \equiv_{\mathrm{ss}} V_{v}^{\prime}(\bmod \lambda)$, then one has $V_{v} \simeq_{\mathrm{ss}} V_{v}^{\prime} .\left[\right.$ In particular, if $V \equiv_{\mathrm{ss}} V^{\prime}(\bmod \lambda)$ as $G_{K}$-representations, then $V_{v} \simeq_{\mathrm{ss}} V_{v}^{\prime}$.]

The constant $C$ can be taken explicitly to be

$$
C:=\max \left\{e^{2} b+1,\left(2\left(\begin{array}{c}
n \\
{[n / 2]}
\end{array}\right) q_{v}^{n b}\right)^{[E: \mathbb{Q}]}\right\},
$$

where $[x]$ denotes the largest integer not exceeding $x$.

As in the Introduction, the meaning of the notations $\equiv_{\mathrm{ss}}$ and $\simeq_{\mathrm{ss}}$ is as follows: we say $V_{v} \equiv_{\mathrm{ss}} V_{v}^{\prime}(\bmod \lambda)$ if, for a choice of $G_{v}$-stable $\mathcal{O}_{E_{\lambda}}$-lattices $T$ and $T^{\prime}$ in $V$ and $V^{\prime}$ respectively, $T / \lambda T$ and $T^{\prime} / \lambda T^{\prime}$ have isomorphic semisimplifications $(T / \lambda T)^{\mathrm{ss}}$ and $\left(T^{\prime} / \lambda T^{\prime}\right)^{\mathrm{ss}}$ as $k_{\lambda}$-linear representations of $G_{v}$. The notation $V \equiv_{\mathrm{ss}} V^{\prime}$ $(\bmod \lambda)$ is defined similarly with the acting group replaced by $G_{K}$. We also say $V_{v} \simeq_{\mathrm{ss}} V_{v}^{\prime}$ if their semisimplifications are isomorphic as $E_{\lambda}$-linear representations of $G_{v}$. 
To state a variant of this theorem, let Conditions (II) be Conditions (I) with $\left(\mathrm{SS}_{v}\right)$ removed; that is, we set

Conditions (II) $:=\left(\operatorname{Int}_{v}\right)+\left(\mathrm{PST}_{u}\right)+\left(\mathrm{HT}_{u}\right)+\left(\mathrm{G}_{u, v}\right)$.

Let $\mathrm{W}_{v}(V)$ denote the multi-set of Weil weights of $V$ (Def. 2.1) considered as a $\mathbb{Q}_{\ell}$-linear representation of $G_{v}$.

Theorem 3.2. For $K, E, n, b, e, v$ as above, the following holds with the same constant $C=C\left([E: \mathbb{Q}], n, b, e, q_{v}\right)$ as in Theorem 3.1: For any prime number $\ell>C$, any places $u$ of $K$ and $\lambda$ of $E$ both lying above $\ell$, and any representations $V$ and $V^{\prime}$ satisfying Conditions (II) with $\mathrm{HT}_{u}(V) \subset[0, b]$, if one has $V_{u} \equiv_{\mathrm{ss}} V_{u}^{\prime}(\bmod \lambda)$ and $V_{v} \equiv_{\mathrm{ss}} V_{v}^{\prime}(\bmod \lambda)$, then one has $\mathrm{W}_{v}(V)=\mathrm{W}_{v}\left(V^{\prime}\right)$. [In particular, if $V \equiv_{\mathrm{SS}} V^{\prime}(\bmod \lambda)$ as $G_{K}$-representations, then $\mathrm{W}_{v}(V)=\mathrm{W}_{v}\left(V^{\prime}\right)$.]

Remark 3.3. If we consider representations of type (W) at all places $v \mid q$ for a fixed prime number $q$ and of Hodge-Tate type at all places $u \mid \ell$, we can prove versions of Theorems 3.1 and 3.2 without assuming "type $(\mathrm{G})$ " but with a larger constant

$$
C^{\prime}:=\max \left\{e^{2} b+1,\left(2\left(\begin{array}{c}
n \\
{[n / 2]}
\end{array}\right) q^{n b[K: \mathbb{Q}] /\left[K_{v}: \mathbb{Q}_{q}\right]}\right)^{[E: \mathbb{Q}]}\right\} .
$$

The proofs are basically the same as in the case of type $(\mathrm{G})$ but use Proposition 2.8 instead of the equality $(\mathrm{G})$ in Definition 2.4 .

The constant $C=C\left([E: \mathbb{Q}], n, b, e, q_{v}\right)$ above depends on the coefficient field $E$. By working modulo $\ell$ rather than modulo $\lambda$, however, we can suppress this dependence as follows:

Theorem 3.4. For any $K, E, n, b, e, v$ as above, there exists a constant $\tilde{C}=$ $\tilde{C}\left(n, b, e, q_{v}\right)$ such that the following holds: For any prime number $\ell>\tilde{C}$, any places $u$ of $K$ and $\lambda$ of $E$ both lying above $\ell$, and any representations $V$ and $V^{\prime}$ satisfying Conditions $(\mathrm{I})$ with $\mathrm{HT}_{u}(V) \subset[0, b]$, if one has $V_{u} \equiv_{\mathrm{ss}} V_{u}^{\prime}(\bmod \lambda)$ and $\operatorname{det}\left(T-\operatorname{Frob}_{v} \mid V_{v}\right) \equiv \operatorname{det}\left(T-\operatorname{Frob}_{v} \mid V_{v}^{\prime}\right)\left(\bmod \ell \mathcal{O}_{E}\right)$, then one has $V_{v} \simeq_{\mathrm{ss}} V_{v}^{\prime}$. [In particular, if $V \equiv_{\mathrm{ss}} V^{\prime}(\bmod \ell)$ as $G_{K}$-representations, then $V \simeq_{\mathrm{ss}} V^{\prime}$ as $G_{v^{-}}^{\prime}$ representations.]

The constant $\tilde{C}$ can be taken explicitly to be

$$
\tilde{C}:=\max \left\{e^{2} b+1,2\left(\begin{array}{c}
n \\
{[n / 2]}
\end{array}\right) q_{v}^{n b}\right\} .
$$

Here, the notation $V \equiv_{\mathrm{ss}} V^{\prime}(\bmod \ell)$ means that $T / \ell T$ and $T^{\prime} / \ell T^{\prime}$ have isomorphic semisimplifications $(T / \ell T)^{\mathrm{ss}}$ and $\left(T^{\prime} / \ell T^{\prime}\right)^{\mathrm{ss}}$ as $k_{\lambda}\left[G_{K}\right]$-modules, where $T$ and $T^{\prime}$ are $\mathcal{O}_{E}$-lattices as before. Note that $T / \ell T$ is a priori an $\left(\mathcal{O}_{E} / \ell \mathcal{O}_{E}\right)\left[G_{K}\right]$ - 
module but we regard it also as a $k_{\lambda}\left[G_{K}\right]$-module via the canonical ring homomorphism $k_{\lambda} \rightarrow \mathcal{O}_{E} / \ell \mathcal{O}_{E}$.

We have the following obvious variant with Conditions (II) (or the modulo $\ell$ version of Theorem 3.2):

Theorem 3.5. For any $K, E, n, b, e, v$ as above, the following holds with the same constant $\tilde{C}=\tilde{C}\left(n, b, e, q_{v}\right)$ as above: For any prime number $\ell>\tilde{C}$, any places $u$ of $K$ and $\lambda$ of $E$ both lying above $\ell$, and any representations $V$ and $V^{\prime}$ satisfying Conditions (II) with $\operatorname{HT}_{u}(V) \subset[0, b]$, if $V_{u} \equiv_{\mathrm{ss}} V_{u}^{\prime}(\bmod \lambda)$ and $\operatorname{det}\left(T-\operatorname{Frob}_{v} \mid V_{v}\right) \equiv$ $\operatorname{det}\left(T-\operatorname{Frob}_{v} \mid V_{v}^{\prime}\right)\left(\bmod \ell \mathcal{O}_{E}\right)$, then $\mathrm{W}_{v}(V)=\mathrm{W}_{v}\left(V^{\prime}\right)$. [In particular, if $V \equiv_{\mathrm{ss}}$ $V^{\prime}(\bmod \ell)$ as $G_{K}$-representations, then $\mathrm{W}_{v}(V)=\mathrm{W}_{v}\left(V^{\prime}\right)$.]

The rest of this section is devoted to the proof of the theorems. We begin with a version of the gap principle:

Lemma 3.6. Let $E, n, v$ be as before, and let $w \in \mathbb{R}_{\geq 0}$ be given. Then there exists a constant $C_{1}=C_{1}\left([E: \mathbb{Q}], n, q_{v}^{w}\right)>0$ such that, for any prime $\ell>C_{1}$ and for any $n$-dimensional $E_{\lambda}$-linear representations $V, V^{\prime}$ of $G_{v}$ which are of type $(\mathrm{W})$, E-integral at $v$ and such that $\Sigma\left(\mathrm{W}_{v}(V)\right), \Sigma\left(\mathrm{W}_{v}\left(V^{\prime}\right)\right)$ are in $\left[0,\left[E_{\lambda}: \mathbb{Q}_{\ell}\right] \cdot w\right]$, the following hold:

(i) If $V \equiv_{\mathrm{ss}} V^{\prime}(\bmod \lambda)$ as $G_{v}$-representations, then $\mathrm{W}_{v}(V)=\mathrm{W}_{v}\left(V^{\prime}\right)$.

(ii) Assume further that $V^{\mathrm{ss}}$ and $\left(V^{\prime}\right)^{\mathrm{ss}}$ are unramified. If $V \equiv_{\mathrm{ss}} V^{\prime}(\bmod \lambda)$ as $G_{v}$-representations, then $V \simeq_{\mathrm{ss}} V^{\prime}$ as $G_{v}$-representations.

The constant $C_{1}$ can be taken explicitly to be

$$
C_{1}:=\left(2\left(\begin{array}{c}
n \\
{[n / 2]}
\end{array}\right) q_{v}^{w / 2}\right)^{[E: \mathbb{Q}]} .
$$

We also have the following modulo $\ell$ version of (ii) above, in which the constant is independent of $[E: \mathbb{Q}]$ :

Lemma 3.7. Let $E, n, v$ be as before, and let $w \in \mathbb{R}_{\geq 0}$ be given. Then there exists a constant $\tilde{C}_{1}=\tilde{C}_{1}\left(n, q_{v}^{w}\right)>0$ such that, for any prime $\ell>C_{1}$ and for any $n$-dimensional $E_{\lambda}$-linear representations $V, V^{\prime}$ of $G_{v}$ such that $V^{\mathrm{ss}},\left(V^{\prime}\right)^{\mathrm{ss}}$ are unramified and which are of type $(\mathrm{W})$, E-integral at $v$ and such that $\Sigma\left(\mathrm{W}_{v}(V)\right), \Sigma\left(\mathrm{W}_{v}\left(V^{\prime}\right)\right)$ are in $\left[0,\left[E_{\lambda}: \mathbb{Q}_{\ell}\right] \cdot w\right]$, the following holds: If $\left.\operatorname{det}\left(T-\operatorname{Frob}_{v} \mid V\right) \equiv \operatorname{det}\left(T-\operatorname{Frob}_{v} \mid V^{\prime}\right)\left(\bmod \ell \mathcal{O}_{E}\right)\right)$, then $V \simeq_{\mathrm{ss}} V^{\prime}$ as $G_{v^{-}}$ representations.

The constant $\tilde{C}_{1}$ can be taken explicitly to be

$$
\tilde{C}_{1}:=2\left(\begin{array}{c}
n \\
{[n / 2]}
\end{array}\right) q_{v}^{w / 2}
$$


As the proofs are similar, we only give a proof of Lemma 3.6.

Proof of Lemma 3.6. Choose a totally ramified extension $K_{v^{\prime}}^{\prime} / K_{v}$ over which $V$ and $V^{\prime}$ become semistable (cf. Lemma 2.3). Let $P(T)=\operatorname{det}\left(T-\operatorname{Frob}_{v^{\prime}} \mid V^{\mathrm{ss}}\right)$ and $P^{\prime}(T)=\operatorname{det}\left(T-\right.$ Frob $\left._{v^{\prime}} \mid\left(V^{\prime}\right)^{\text {ss }}\right)$ be the characteristic polynomials (taken as $E_{\lambda}$-linear representations) of the Frobenius Frob $_{v^{\prime}}$ at $v^{\prime}$ acting on the semisimplifications $V^{\text {ss }}$ and $\left(V^{\prime}\right)^{\text {ss }}$, respectively. By assumption, they have coefficients in $\mathcal{O}_{E}$. By assumption on the weights, for any embedding $E \hookrightarrow \mathbb{C}$, the terms of $T^{n-i}$ have coefficients of absolute value $\leq\left(\begin{array}{c}n \\ i\end{array}\right) q_{v}^{w / 2}$ (note that $\Sigma\left(\mathrm{W}_{v}(V)\right)$ is the sum of the Weil weights of $V$ as a $\mathbb{Q}_{\ell}$-linear representation, and hence the sum of the Weil weights of the roots of $P(T)$ is in $[0, w])$. Set $C_{1}:=\left(2 \max _{0 \leq i \leq n}\left(\begin{array}{c}n \\ i\end{array}\right) q_{v}^{w / 2}\right)^{[E: \mathbb{Q}]}=$ $\left(2\left(\begin{array}{c}n \\ {[n / 2]}\end{array}\right) q_{v}^{w / 2}\right)^{[E: \mathbb{Q}]}$. Then if $\ell>C_{1}$, we have

$$
\begin{aligned}
& V \equiv_{\mathrm{ss}} V^{\prime}(\bmod \lambda) \text { as } G_{v} \text {-representations } \\
& \qquad P(T) \equiv P^{\prime}(T)(\bmod \lambda) \Leftrightarrow P(T)=P^{\prime}(T) .
\end{aligned}
$$

Here, the last equivalence follows from the next lemma. This implies that $\mathrm{W}_{v}(V)=$ $\mathrm{W}_{v}\left(V^{\prime}\right)$. If $V^{\mathrm{ss}}$ and $\left(V^{\prime}\right)^{\mathrm{ss}}$ are unramified, then they are determined by the actions of Frob $_{v}$, and hence the equality $P(T)=P^{\prime}(T)$ is equivalent to $V \simeq_{\mathrm{ss}} V^{\prime}$.

Lemma 3.8. Let $a$ be a non-zero integer of $E$, and $C_{0}$ a real number $>0$. If $a \equiv 0(\bmod \lambda)\left(\right.$ resp. $\left.a \equiv 0\left(\bmod \ell \mathcal{O}_{E}\right)\right)$ and $|\iota(a)| \leq C_{0}$ for any embedding $\iota: E \hookrightarrow \mathbb{C}$, then $\ell \leq C_{0}^{[E: \mathbb{Q}] / f_{\lambda}}\left(\right.$ resp. $\left.\ell \leq C_{0}\right)$.

Proof. If $\lambda \mid a$ (resp. $\ell \mid a$ ) in $\mathcal{O}_{E}$, then by taking the norm $N: E^{\times} \rightarrow \mathbb{Q}^{\times}$, we have $\ell^{f_{\lambda}} \leq|N(a)|$ (resp. $\ell^{[E: \mathbb{Q}]} \leq|N(a)|$ ). If $|\iota(a)| \leq C_{0}$, then by taking the norm (or product over all $\iota$ ), we have $|N(a)| \leq C_{0}^{[E: \mathbb{Q}]}$. The required inequality follows from these two inequalities.

We need one more lemma:

Lemma 3.9. Let $G$ be a profinite group and $T, T^{\prime}$ be free $\mathcal{O}_{E_{\lambda}}$-modules on which $G$ acts continuously and $\mathcal{O}_{E_{\lambda}}$-linearly. Let $(T / \lambda T)^{\mathrm{ss}}$ and $(T / \ell T)^{\mathrm{ss}}$ be the semisimplifications of $T / \lambda T$ and $T / \ell T$ as $k_{\lambda}[G]$-modules, respectively. Let e be the ramification index of $E_{\lambda} / \mathbb{Q}_{\ell}$. Then:

(i) $(T / \ell T)^{\mathrm{ss}}$ is isomorphic to the direct sum of e copies of $(T / \lambda T)^{\mathrm{ss}}$.

(ii) If $(T / \lambda T)^{\mathrm{ss}} \simeq\left(T^{\prime} / \lambda T^{\prime}\right)^{\mathrm{ss}}$, then $(T / \ell T)^{\mathrm{ss}} \simeq\left(T^{\prime} / \ell T^{\prime}\right)^{\mathrm{ss}}$.

Proof. Part (ii) follows from (i) immediately. To prove (i), consider the filtration

$$
T / \ell T=T / \lambda^{e} T \supset \lambda T / \lambda^{e} T \supset \cdots \supset \lambda^{e} T / \lambda^{e} T=0 .
$$


Then "multiplication by $\lambda$ " (where $\lambda$ is identified with a uniformizer at $\lambda$ ) induces isomorphisms $\lambda^{i} T / \lambda^{i+1} T \rightarrow \lambda^{i+1} T / \lambda^{i+2} T$ of the graded quotients as $k_{\lambda}[G]$ modules. It then follows that $(T / \ell T)^{\mathrm{ss}} \simeq\left((T / \lambda T)^{\mathrm{ss}}\right)^{\oplus e}$.

Now we can prove the theorems. We only prove Theorems 3.1 and 3.2, the proofs of Theorems 3.4 and 3.5 being similar.

Let $C=\max \left\{e^{2} b+1,\left(2\left(\begin{array}{c}n \\ {[n / 2]}\end{array}\right) q_{v}^{n b}\right)^{[E: \mathbb{Q}]}\right\}$, as in Theorem 3.1. Choose a finite totally ramified extension $\left(K_{u}\right)^{\prime} / K_{u}$, with absolute ramification index $e^{2}$, over which $V$ and $V^{\prime}$ become semistable (cf. Lemma 2.3). If $\ell>C$, then $e^{2} b<\ell-1$. Take a finite extension $K^{\prime}$ of $K$ and a place $u^{\prime} \mid u$ of $K^{\prime}$ such that the completion of $K^{\prime}$ at $u^{\prime}$ is $\left(K_{u}\right)^{\prime}$. By assumption, we have $\operatorname{HT}_{u^{\prime}}(V) \subset[0, b]$. Then by Theorem 2.6(i), we have $\mathrm{TI}_{u^{\prime}}(V) \subset[0, b]$. The same holds for $V^{\prime}$, since $\mathrm{TI}_{u^{\prime}}(V)=\mathrm{TI}_{u^{\prime}}\left(V^{\prime}\right)$ by the assumption $V_{u} \equiv_{\mathrm{ss}} V_{u}^{\prime}(\bmod \lambda)$ (Note that, by Lemma 3.9, we also have $V_{u} \equiv_{\mathrm{ss}}$ $V_{u}^{\prime}(\bmod \ell)$ as $\mathbb{F}_{\ell}\left[G_{u}\right]$-modules, where $V$ and $V^{\prime}$ are now regarded as $\mathbb{Q}_{\ell}$-linear representations, so that the definition of $\mathrm{TI}_{u}$ and Theorem 2.6 are applicable). Now we recall that $V$ and $V^{\prime}$ are of type (G). By Theorem 2.6(ii), we have

$$
\Sigma\left(\mathrm{TI}_{u^{\prime}}(V)\right)=\Sigma\left(\operatorname{HT}_{u^{\prime}}(V)\right)=\Sigma\left(\operatorname{HT}_{u}(V)\right)=\frac{1}{2} \Sigma\left(\mathrm{W}_{v}(V)\right),
$$

and these are also equal to

$$
\Sigma\left(\operatorname{TI}_{u^{\prime}}\left(V^{\prime}\right)\right)=\Sigma\left(\operatorname{HT}_{u^{\prime}}\left(V^{\prime}\right)\right)=\Sigma\left(\operatorname{HT}_{u}\left(V^{\prime}\right)\right)=\frac{1}{2} \Sigma\left(\mathrm{W}_{v}\left(V^{\prime}\right)\right) .
$$

Since $\operatorname{HT}_{u}(V) \subset[0, b]$, these are bounded by $\left[E_{\lambda}: \mathbb{Q}_{\ell}\right] \cdot n b$. In particular, the total weights $\Sigma\left(\mathrm{W}_{v}(V)\right)$ and $\Sigma\left(\mathrm{W}_{v}\left(V^{\prime}\right)\right)$ are $\leq\left[E_{\lambda}: \mathbb{Q}_{\ell}\right] \cdot 2 n b$. By Lemma 3.6(i) (resp. (ii)), the assumption $V_{v} \equiv_{\mathrm{ss}} V_{v}^{\prime}(\bmod \lambda)$ implies that $\mathrm{W}_{v}(V)=\mathrm{W}_{v}\left(V^{\prime}\right)$ (resp. $\left.V_{v} \simeq_{\mathrm{ss}} V_{v}^{\prime}\right)$ if $\ell>\left(2\left(\begin{array}{c}n \\ {[n / 2]}\end{array}\right) q_{v}^{n b}\right)^{[E: \mathbb{Q}]}$.

\section{$\S 4$. Corollaries}

Here we give several corollaries of Theorem 3.2, which are motivated by a conjecture of Rasmussen and Tamagawa ([23]; see also [4], [20], [21] and [24]). The conjecture says that, for a prime number $\ell$ large enough, there does not exist an abelian variety $A$ of a given dimension over a given number field with good reduction outside $\ell$ and such that the Galois representation on $A[\ell]$ is Borel whose diagonal components are powers of the modulo $\ell$ cyclotomic character. The purpose of this section is to generalize such a non-existence statement for abelian varieties to one for algebraic varieties in terms of étale cohomology groups by applying one of our main theorems of $\S 3$. This is a sort of formalization of the method of [20] and [24]. In these papers, the Rasmussen-Tamagawa conjecture was proved under the assumption that the abelian varieties have semistable reduction everywhere. An advantage of our main results is that we can consider 
not only semistable representations associated with abelian varieties but also a certain class of potentially semistable representations associated with algebraic varieties.

The notations $\left(K, E, n, b, e, v, u, \ell, \lambda, C=C\left([E: \mathbb{Q}], n, b, e, q_{v}\right), \ldots\right)$ are the same as in Theorem 3.2. In this section, $V=V_{X}^{r}$ will be the $E_{\lambda}$-linear dual $H_{\mathrm{et}}^{r}\left(X_{\bar{K}}, E_{\lambda}\right)^{*}$ of the $r$-th $\lambda$-adic étale cohomology group, where $X$ is a smooth proper variety over $K$, and $X_{\bar{K}}$ denotes its base extension to $\bar{K}$. We set $\bar{V}=$ $\bar{V}_{X}^{r}:=T / \lambda T$, choosing a $G_{K}$-stable $\mathcal{O}_{E_{\lambda}}$-lattice $T$ in $V$, and let $\bar{V}^{\text {ss }}=\bar{V}_{X}^{r \text {,ss }}$ be its semisimplification as a $k_{\lambda}\left[G_{K}\right]$-module (the isomorphism class of $\bar{V}_{X}^{r \text {,ss }}$ does not depend on the choice of $T$ ).

To state the first corollary, we make the following hypothesis on $\bar{V}^{\text {ss: }}$

Hypothesis (H). Each simple factor $\bar{W}$ of $\bar{V}^{\text {ss }}$ lifts to an $E_{\lambda}$-linear representation $W$ of $G_{K}$ of the form $H_{\mathrm{et}}^{s}\left(Y_{\bar{K}}, E_{\lambda}\right)^{*}$ which is semistable at all $u \mid \ell$, and $\operatorname{HT}_{u}(W) \subset$ $[0, \ell-2]$, where $Y$ is a proper smooth variety over $K$ and $s$ is some non-negative integer.

Corollary 4.1. For any prime $\ell>C$, any odd integer $r$ with $1 \leq r \leq b$, any places $u$ of $K$ and $\lambda$ of $E$ both lying above $\ell$, and any smooth proper variety $X$ which has the $r$-th Betti number $\leq n$, has potentially good reduction at $v$, and has semistable reduction at $u \mid \ell$, if $(\mathrm{H})$ is true for $\bar{V}_{X}^{r, \mathrm{ss}}$, then none of the simple factors of $\bar{V}_{X}^{r, \mathrm{ss}}$ are of odd dimension.

Proof. Note first that, if $s$ is odd, then $H_{\mathrm{et}}^{s}\left(Y_{\bar{K}}, E_{\lambda}\right)$ has even dimension by (GAGA and) Hodge theory. Now, let $\bar{W}_{1}, \ldots, \bar{W}_{k}$ be the simple factors of $\bar{V}^{\text {ss }}$. By $(\mathrm{H})$, each $\bar{W}_{i}$ lifts to a geometric $W_{i}$ with $\mathrm{HT}_{u}\left(W_{i}\right) \subset[0, \ell-2]$. If one of the $W_{i}$ has odd dimension, then it must have even weight, while $V$ has odd weight $r$, since $X$ has potentially good reduction at $v$. Thus the corollary follows from Theorem 3.2 by putting $V^{\prime}:=W_{1} \oplus \cdots \oplus W_{k}$.

As a special case where Hypothesis $(\mathrm{H})$ holds, we have:

Corollary 4.2. For any prime number $\ell>C$, any odd integer $r$ with $1 \leq r \leq b$, any places $u$ of $K$ and $\lambda$ of $E$ both lying above $\ell$, and any smooth proper variety $X$ over $K$ which has $r$-th Betti number $\leq n$, has potentially good reduction at $v$, and has semistable reduction at $u$, the Galois representation on $\bar{V}_{X}^{r, s s}$ is not the sum of integral powers of modulo $\ell$ cyclotomic characters.

In fact, we can generalize this a bit as follows. Let $\chi$ and $\bar{\chi}$ denote respectively the $\ell$-adic and modulo $\ell$ cyclotomic characters of $G_{K}$. 
Corollary 4.3. Assume $E$ contains the $e^{2}$-th roots of unity. Then for any prime number $\ell>C$ such that $\ell \equiv 1\left(\bmod e^{2}\right)$, any odd integer $r$ with $1 \leq r \leq b$, any places $u$ of $K$ and $\lambda$ of $E$ both lying above $\ell$, and any smooth proper variety $X$ over $K$ which has $r$-th Betti number $\leq n$, has potentially good reduction at $v$, and acquires semistable reduction over a finite extension $K_{u^{\prime}}^{\prime} / K_{u}$ with absolute ramification index $e\left(K_{u^{\prime}}^{\prime} / \mathbb{Q}_{\ell}\right)$ dividing e, the Galois representation $\bar{V}_{X}^{r, \mathrm{ss}}$ is not the sum of characters of $G_{K}$ of the form $\bar{\varepsilon}_{i} \bar{\chi}^{b_{i}}$, where $\bar{\varepsilon}_{i}: G_{K} \rightarrow k_{\lambda}^{\times}$are characters unramified at $u$ and of finite order dividing the order of the group of roots of unity in $E$, and $b_{i}$ are integers.

Proof. Suppose $X$ has semistable reduction over $K_{u^{\prime}}^{\prime}$ with $e\left(K_{u^{\prime}}^{\prime} / \mathbb{Q}_{\ell}\right) \mid e$. We may assume $e\left(K_{u^{\prime}}^{\prime} / \mathbb{Q}_{\ell}\right)=e$. Suppose $\bar{V}^{\text {ss }}$ is the sum of the characters $\bar{\varepsilon}_{i} \bar{\chi}^{b_{i}}$ as above. Then the action of the tame inertia group $I_{u^{\prime}}^{\text {tame }}$ at $u^{\prime}$ on the $i$-th factor is via $\bar{\chi}^{b_{i}}$, which equals $\theta^{e b_{i}}$, where $\theta$ is the fundamental character of $I_{u^{\prime}}^{\text {tame }}$ of level 1 ([30, $\S 1.8$, Prop. 8]). By Theorem 2.6(i), we have $e b_{i} \equiv c_{i}(\bmod \ell-1)$ with $0 \leq c_{i} \leq e b$. Since $e^{2} \mid \ell-1$, we have $b_{i}=b_{0 i}+\frac{\ell-1}{e^{2}} j$ with $0 \leq b_{0 i} \leq b$ and $0 \leq j<e^{2}$. Set $\bar{\kappa}:=\bar{\chi}^{(\ell-1) / e^{2}}$ and let $\kappa: G_{K} \rightarrow E_{\lambda}^{\times}$be its Teichmüller lift. Since the $e^{2}$-th power of $\kappa$ is trivial, it takes values in $E^{\times}$. Similarly, the Teichmüller lift $\varepsilon_{i}$ of $\bar{\varepsilon}_{i}$ has also values in $E^{\times}$. Now each character $\bar{\varepsilon}_{i} \bar{\chi}^{b_{i}}=\bar{\varepsilon}_{i} \bar{\kappa}^{j} \bar{\chi}^{b_{0 i}}$ lifts to the character $\varepsilon_{i} \kappa^{j} \chi^{b_{0 i}}: G_{K} \rightarrow E_{\lambda}^{\times}$, or to the 1-dimensional $E_{\lambda}$-linear $E$-integral geometric representation $E_{\lambda}\left(\varepsilon_{i} \kappa^{j}\right) \otimes_{\mathbb{Q}_{\ell}} \mathbb{Q}_{\ell}\left(b_{0 i}\right)$, where $E_{\lambda}\left(\varepsilon_{i} \kappa^{j}\right)$ is the twist of the trivial representation $E_{\lambda}$ by the finite character $\varepsilon_{i} \kappa^{j}$, and $\mathbb{Q}_{\ell}\left(b_{0 i}\right)$ denotes the $b_{0 i}$-th Tate twist. Let $V^{\prime}$ be the direct sum of these representations. By Theorem 3.2, we have $\mathrm{W}_{v}(V)=\mathrm{W}_{v}\left(V^{\prime}\right)$, but $\mathrm{W}_{v}(V)=\{r, \ldots, r\}$ (since $X$ has potentially good reduction at $v$ ) while $\mathrm{W}_{v}\left(V^{\prime}\right)=\left\{2 b_{01}, \ldots, 2 b_{0 n}\right\}$, which is a contradiction if $r$ is odd.

Specializing further, we have:

Corollary 4.4. Let $K=\mathbb{Q}$. Assume $E$ contains the $e^{2}$-th roots of unity. Then for any prime number $\ell>C$ such that $\ell \equiv 1\left(\bmod e^{2}\right)$, for any odd integer $r$ with $1 \leq r \leq b$, and for any smooth proper variety $X$ over $\mathbb{Q}$ which has $r$-th Betti number $\leq n$, has good reduction outside $\ell$ and acquires semistable reduction over a finite extension $K_{u^{\prime}}^{\prime} / \mathbb{Q}_{\ell}$ with absolute ramification index $e\left(K_{u^{\prime}}^{\prime} / \mathbb{Q}_{\ell}\right)$ dividing e, the Galois representation on $\bar{V}_{X}^{r}$ is not Borel.

Here, we say that the representation $\bar{V}$ is Borel if the action of $G_{\mathbb{Q}}$ is given by upper-triangular matrices with respect to a suitable $k_{\lambda}$-basis of $\bar{V}$.

Proof. Indeed, if it is Borel, its semisimplification is a sum of characters which are unramified outside $\ell$ by assumption. Since the base field is $\mathbb{Q}$, they are powers 
of the modulo $\ell$ cyclotomic character. Now the result follows from the previous corollary.

\section{$\S 5$. Congruences of modular forms}

We use the same notations as in the Introduction, except that we always suppose $K=\mathbb{Q}$ and write $q$ for $q_{v}$ in this section. We put $\varphi(N)=\#(\mathbb{Z} / N \mathbb{Z})^{\times}$for any positive integer $N$ and denote by $\overline{\mathbb{Z}}$ the integer ring of $\overline{\mathbb{Q}}$. The goal of this section is to give a proof of the following congruence result on the Fourier coefficients of modular forms. For any integers $k, N \geq 1$ and a character $\epsilon:(\mathbb{Z} / N \mathbb{Z})^{\times} \rightarrow \mathbb{C}^{\times}$, let $S_{k}(N, \epsilon)$ denote the $\mathbb{C}$-vector space of cusp forms of weight $k$, level $N$ and Nebentypus character $\epsilon$. We denote by $N_{\epsilon}$ the conductor of $\epsilon$. For a normalized Hecke eigenform $f(z)=\sum_{n=1}^{\infty} a_{n}(f) e^{2 \pi i n z} \in S_{k}(N, \epsilon)$, integers $i, j$ and a prime number $\ell$, consider the following condition on the Fourier coefficients $a_{p}(f)$ of $f$ :

$\left(\mathrm{C}_{i, j ; \ell}\right) \quad a_{p}(f) \equiv p^{i}+p^{j}(\bmod \ell \overline{\mathbb{Z}}) \quad$ for all but finitely many primes $p \nmid \ell N$.

Such congruences can be interpreted in terms of the Galois representation $\rho_{f, \lambda}$ associated with $f$, where $\lambda$ is a place above $\ell$ of the Hecke field of $f$. For example, the congruence $\left(\mathrm{C}_{i, j ; \ell}\right)$ holds if $\rho_{f, \lambda} \equiv_{\mathrm{ss}} \chi^{i} \oplus \chi^{j}(\bmod \ell)$, where $\chi$ is the $\ell$-adic cyclotomic character. We say, for the moment, that a prime $\ell$ is exceptional for $f$ if the image of the residual representation of $\rho_{f, \lambda}$ does not contain $\mathrm{SL}_{2}\left(k_{\lambda}\right)$ for some $\lambda \mid \ell$ (cf. [31, §3.1] and [19, §3]). For fixed $k$ and $N$, it is well known (cf. e.g. [31, Thm. 10] and [18, Introduction]) that there are only finitely many exceptional primes, and a fortiori finitely many primes $\ell$ for which $\left(\mathrm{C}_{i, j ; \ell}\right)$ holds for some $i, j$ and $f \in S_{k}(N, \epsilon)$. Until recently, however, the situation has not been very clear when we let $k$ and $N$ vary; as for recent works, see [15] for the case of modular Abelian varieties (i.e., the case of weight $k=2$ ) and [2] for the case of modular forms on $\Gamma_{0}(N)$ with fixed level $N$. In this vein, we show the following by using Theorem 3.4:

Theorem 5.1. Fix a prime number $q$. For any integer $k \geq 1$, any prime $\ell>4 q^{2(k-1)}$, any integer $N$ such that $q \nmid N$ and $\ell^{2} \nmid N$, any character $\epsilon$ : $(\mathbb{Z} / N \mathbb{Z})^{\times} \rightarrow \mathbb{C}^{\times}$with $\ell \nmid \varphi\left(N_{\epsilon}\right)$, and any normalized Hecke eigenform $f \in S_{k}(N, \epsilon)$, we have the following:

(i) The condition $\left(\mathrm{C}_{i, j ; \ell}\right)$ can hold only if $i \equiv j \equiv(k-1) / 2(\bmod \ell-1)$.

(ii) The condition $\left(\mathrm{C}_{i, j ; \ell}\right)$ holds for no $i$ and $j$ if either $k=1, k$ is even, or $\ell \nmid N$.

We begin by proving a lemma. For any $f$ as in the theorem, we denote by $E=\mathbb{Q}_{f}$ the field obtained by adjoining all Fourier coefficients of $f$ to $\mathbb{Q}$, which is 
a finite extension of $\mathbb{Q}$. We regard $\epsilon$ as a character with values in $\mathcal{O}_{E}^{\times}$. Denote by $\bar{\epsilon}$ (resp. $\left.\bar{\epsilon}_{\lambda}\right)$ the composite $(\mathbb{Z} / N \mathbb{Z})^{\times} \stackrel{\epsilon}{\rightarrow} \mathcal{O}_{E}^{\times} \stackrel{\bmod \ell}{\longrightarrow}\left(\mathcal{O}_{E} / \ell \mathcal{O}_{E}\right)^{\times}\left(\operatorname{resp} .(\mathbb{Z} / N \mathbb{Z})^{\times} \stackrel{\epsilon}{\rightarrow}\right.$ $\left.\mathcal{O}_{E}^{\times} \stackrel{\bmod \lambda}{\longrightarrow}\left(\mathcal{O}_{E} / \lambda \mathcal{O}_{E}\right)^{\times}\right)$. Let

$$
\rho_{f, \lambda}: G_{\mathbb{Q}} \rightarrow \mathrm{GL}_{E_{\lambda}}\left(V_{f, \lambda}\right)
$$

be the 2-dimensional $E_{\lambda}$-linear representation of $G_{\mathbb{Q}}$ associated with $f$ by Deligne [8] and Deligne-Serre [10]. Thus if $p \nmid \ell N$, then $V_{f, \lambda}$ is unramified at $p$ and one has

$$
\operatorname{det}\left(T-\operatorname{Frob}_{p} \mid V_{f, \lambda}\right)=T^{2}-a_{p}(f) T+\epsilon(p) p^{k-1} .
$$

In particular, it is $E$-integral at $p$ in the sense of Definition 2.2. One has $\mathrm{W}_{p}\left(V_{f, \lambda}\right)=$ $\{(k-1) / 2, \ldots,(k-1) / 2\}$ (with multiplicity $2\left[E_{\lambda}: \mathbb{Q}_{\ell}\right]$ ). It is crystalline (resp. semistable) at $\ell$ if $\ell \nmid N$ (resp. $\left.\ell^{2} \nmid N\right)$.

Lemma 5.2. Suppose $\ell>2$. Let $k, N \geq 1$ be two integers. Let $\epsilon:(\mathbb{Z} / N \mathbb{Z})^{\times} \rightarrow \mathbb{C}^{\times}$ be a character such that $\ell \nmid \varphi\left(N_{\epsilon}\right)$. Suppose that a normalized Hecke eigenform $f \in S_{k}(N, \epsilon)$ satisfies the condition $\left(\mathrm{C}_{i, j ; \ell}\right)$ for some $i, j$. Then $\bar{\epsilon}$ has values in the canonical image of $\mathbb{F}_{\ell}^{\times}$in $\left(\mathcal{O}_{E} / \ell \mathcal{O}_{E}\right)^{\times}$. Moreover, the following holds:

(i) $\bar{\epsilon}(x(\bmod N))=x^{i+j-(k-1)}(\bmod \ell)$ for any $x$ prime to $N$.

(ii) If $\ell \nmid N$, then $i+j \equiv k-1(\bmod \ell-1)$ and $\bar{\epsilon}=1$.

Proof. By assumption, we have $\operatorname{Tr}\left(\operatorname{Frob}_{p} \mid V_{f, \lambda}\right) \equiv p^{i}+p^{j}\left(\bmod \ell \mathcal{O}_{E}\right)$ for all but finitely many $p \nmid \ell N$. In particular,

$$
\rho_{f, \lambda} \equiv_{\mathrm{ss}} \chi^{i} \oplus \chi^{j}(\bmod \lambda)
$$

as $k_{\lambda}$-linear representations of $G_{\mathbb{Q}}$ (this holds because $\ell>2=\operatorname{dim} \rho_{f, \lambda}$; see, e.g., [21, Lemma 2.10]), and then we also have $\epsilon(p) p^{k-1} \equiv p^{i+j}(\bmod \lambda)$. Hence we see that

$$
\bar{\epsilon}_{\lambda}(x(\bmod N))=x^{i+j-(k-1)}(\bmod \lambda)
$$

for any $\lambda \mid \ell$ and any integer $x$ prime to $N$.

(i) Since the kernel of the projection $\left(\mathcal{O}_{E} / \ell \mathcal{O}_{E}\right)^{\times} \rightarrow \prod_{\lambda \mid \ell}\left(\mathcal{O}_{E} / \lambda \mathcal{O}_{E}\right)^{\times}$has $\ell$-power order, if $\ell \nmid \varphi\left(N_{\epsilon}\right)$, then the homomorphism $\prod_{\lambda \mid \ell} \bar{\epsilon}_{\lambda}:(\mathbb{Z} / N \mathbb{Z})^{\times} \rightarrow$ $\prod_{\lambda \mid \ell}\left(\mathcal{O}_{E} / \lambda \mathcal{O}_{E}\right)^{\times}$lifts uniquely to a homomorphism $(\mathbb{Z} / N \mathbb{Z})^{\times} \rightarrow\left(\mathcal{O}_{E} / \ell \mathcal{O}_{E}\right)^{\times}$, which is $\bar{\epsilon}$. According to (5.2), it is given by

$$
\bar{\epsilon}(x(\bmod N))=x^{i+j-(k-1)}\left(\bmod \ell \mathcal{O}_{E}\right)
$$

for any integer $x$ prime to $N$. 
(ii) Suppose $\ell \nmid N$. Then (5.3) must hold for $x=\ell$, which is possible only if $i+j \equiv k-1(\bmod \ell-1)$. In particular, we obtain $\bar{\epsilon}=1$.

Proof of Theorem 5.1. (i) Suppose $\ell \nmid \varphi(N)$ and $\ell^{2} \nmid N$. Then $\rho_{f, \lambda}$ is semistable at $\ell$. By assumption, we have $\operatorname{Tr}\left(\operatorname{Frob}_{q} \mid V_{f, \lambda}\right) \equiv q^{i}+q^{j}\left(\bmod \ell \mathcal{O}_{E}\right)$. Combining this with Lemma 5.2(i), we obtain $\operatorname{det}\left(T-\operatorname{Frob}_{q} \mid V_{f, \lambda}\right) \equiv \operatorname{det}\left(T-\operatorname{Frob}_{q} \mid \chi^{i} \oplus \chi^{j}\right)$ $\left(\bmod \ell \mathcal{O}_{E}\right)$. We also have the congruence $(5.1)$. Therefore, if $\ell>4 q^{2(k-1)}$, it follows from Theorem 3.4 (applied with $V^{\prime}=\chi^{i^{\prime}} \oplus \chi^{j^{\prime}}$, where $i^{\prime}, j^{\prime}$ are integers in $[0, \ell-2]$ such that $\left.i^{\prime} \equiv i, j^{\prime} \equiv j(\bmod \ell-1)\right)$ that $\rho_{f, \lambda} \simeq_{\mathrm{ss}} \chi^{i^{\prime}} \oplus \chi^{j^{\prime}}$ as $E_{\lambda}$-linear representations of the decomposition group $G_{q}$ of $q$. Looking at the Weil weights, we obtain $i \equiv j \equiv(k-1) / 2(\bmod \ell-1)$.

(ii) If $k$ is even, then the impossibility of $\left(\mathrm{C}_{i, j ; \ell}\right)$ follows from (i).

If $k=1$ and the congruence condition $\left(\mathrm{C}_{i, j ; \ell}\right)$ holds, then (i) together with (5.1) implies that $\bar{\rho}_{f, \lambda}:=\rho_{f, \lambda}(\bmod \lambda)$ is unipotent and, in particular, $\operatorname{Im}\left(\bar{\rho}_{f, \lambda}\right)$ is an $\ell$-group. On the other hand, if $k=1$, then by [10], $\operatorname{Im}\left(\rho_{f, \lambda}\right)$ is finite and its image in $\mathrm{PGL}_{2}\left(\mathcal{O}_{E_{\lambda}}\right)$ is either dihedral, $A_{4}, S_{4}$ or $A_{5}$. Since the kernel of the reduction map $\mathrm{GL}_{2}\left(\mathcal{O}_{E_{\lambda}}\right) \rightarrow \mathrm{GL}_{2}\left(k_{\lambda}\right)$ is pro- $\ell$, the representation $\bar{\rho}_{f, \lambda}$ cannot be unipotent if $\ell \geq 3$.

Finally, assume $\ell \nmid N$. Then $\rho_{f, \lambda}$ is crystalline at $\ell$, and thus the FontaineLaffaille theory (cf. [13, Thm. 5.3(iii)]) implies that the tame inertia weights and the Hodge-Tate weights of $\rho_{f, \lambda}$ coincide with each other. Hence it follows from (5.1) that $\{i, j\} \equiv\{0, k-1\}(\bmod \ell-1)$. Since $\ell>k$, we obtain $\{(k-1) / 2,(k-1) / 2\}=$ $\{0, k-1\}$, which is impossible unless $k=1$.

\section{Acknowledgements}

The second-named author thanks Eknath Ghate for his invitation to TIFR, Mumbai, and his interest in this work, which motivated us to write down the results; both the authors are grateful to him for his useful comments on the first version of this paper. The authors thank Tetsushi Ito and Yoichi Mieda for their useful information on $\ell$-adic étale cohomology. Thanks are also due to the referee for detailed comments which greatly improved the exposition of the paper. This work is supported in part by JSPS KAKENHI 25·173, JSPS KAKENHI 22540024 and Adaptable and Seamless Technology Transfer Program through target-driven R\&D, JST.

\section{References}

[1] P. Berthelot, Altérations de variétés algébriques (d'après A. J. de Jong), in Séminaire Bourbaki, 1995/96, exp. 815, Astérisque 241 (1997), 273-311. Zbl 0924.14007 MR 1472543 
[2] N. Billerey and L. V. Dieulefait, Explicit large image theorems for modular forms, J. London Math. Soc. 89 (2014), 499-523.

[3] N. Bourbaki, Éléments de mathématique. Algèbre. Chapitre 8. Modules et anneaux semisimples, 2nd rev. ed., Springer, Berlin, 2012. Z Zbl 1245.16001 MR 3027127

[4] A. Bourdon, A uniform version of a finiteness conjecture for CM elliptic curves, arXiv:1305.5241 (2013).

[5] X. Caruso, Représentations semi-stables de torsion dans le case $e r<p-1$, J. Reine Angew. Math. 594 (2006), 35-92. Zbl 1134.14013 MR 2248152

[6] X. Caruso and D. Savitt, Polygones de Hodge, de Newton et de l'inertie modérée des représentations semi-stables, Math. Ann. 343 (2009), 773-789. Zbl 1248.11092 MR 2471600

[7] A. J. de Jong, Smoothness, semi-stability and alterations, Publ. Math. IHES 83 (1996), 51-93. Zbl 0916.14005 MR 1423020

[8] P. Deligne, Formes modulaires et représentations $\ell$-adiques, in Séminaire Bourbaki, 1968/69, exp. 355, Lecture Notes in Math. 175, Springer, Berlin, 1971, 139-172. Zbl 0206.49901 MR 3077124

[9] , La conjecture de Weil, II, Publ. Math. IHES 52 (1980), 137-252. Zbl 0456.14014 MR 0601520

[10] P. Deligne and J.-P. Serre, Formes modulaires de poids 1, Ann. Sci. École Norm. Sup. 7 (1974), 507-530. Zbl 0321.10026 MR 0379379

[11] G. Faltings, p-adic Hodge theory, J. Amer. Math. Soc. 1 (1988), 255-299. Z Zbl 0764.14012 MR 0924705

[12] J.-M. Fontaine, Représentations $p$-adiques semi-stables, in Périodes p-adiques (Bures-surYvette, 1988), Astérisque 223 (1994), 113-184. Zbl 0865.14009 MR 1293972

[13] J.-M. Fontaine and G. Laffaille, Construction de représentations p-adiques, Ann. Sci. École Norm. Sup. 15 (1982), 547-608. Zbl 0579.14037 MR 0707328

[14] J.-M. Fontaine and B. Mazur, Geometric Galois representations, in: Elliptic curves, modular forms, and Fermat's Last Theorem, J. Coates and S.-T. Yau (eds.), Int. Press, Cambridge, MA, 1995, 41-78 in the 1st ed. (190-227 in the 2nd ed.). Zbl 0839.14011 MR 1363495

[15] E. Ghate and P. Parent, On uniform large Galois images for modular abelian varieties, Bull. London Math. Soc. 44 (2012), 1169-1181. Zbl 06114743 MR 3007649

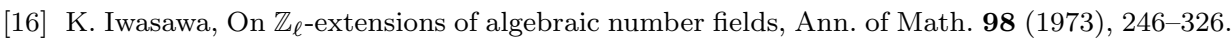
Zbl 0285.12008 MR 0349627

[17] _ Local class field theory, Oxford Univ. Press, New York, 1986. Z Zbl 0604.12014 MR 0863740

[18] I. Kiming and H. Verrill, On modular $\bmod \ell$ Galois representations with exceptional images, J. Number Theory 110 (2005), 236-266. Z Zbl 1068.11037 MR 2122608

[19] K. Ono, Congruences on the Fourier coefficients of modular forms on $\Gamma_{0}(N)$, in: The Rademacher legacy to mathematics (University Park, PA, 1992), Contemp. Math. 166, Amer. Math. Soc., Providence, RI, 1994, 93-105. Zbl 0812.11029 MR 1284053

[20] Y. Ozeki, Non-existence of certain Galois representations with a uniform tame inertia weight, Int. Math. Res. Notices 2011, 2377-2395. Zbl 1231.11059 MR 2806583

[21] Non-existence of certain CM abelian varieties with prime power torsion, Tohoku Math. J. 65 (2013), 357-371. Zbl 06237267 MR 3102540

[22] M. Rapoport and Th. Zink, Über die lokale Zetafunktion von Shimuravarietäten. Monodromiefiltration und verschwindende Zyklen in ungleicher Charakteristik, Invent. Math. 68 (1982), 21-101. Zbl 0498.14010 MR 0666636 
[23] C. Rasmussen and A. Tamagawa, A finiteness conjecture on Abelian varieties with constrained prime power torsion, Math. Res. Lett. 15 (2008), 1223-1231. Zbl 1182.11027 MR 2470396

[24] _ Arithmetic of abelian varieties with constrained torsion, arXiv:1302.1477 (2013).

[25] T. Saito, Weight spectral sequences and independence of $\ell$, J. Inst. Math. Jussieu 2 (2003), 583-634. Zbl 1084.14027 MR 2006800

[26] The second Stiefel-Whitney classes of $\ell$-adic cohomology, J. Reine Angew. Math. 681 (2013), 101-147. Zbl 06211851

[27] S. Sen, Lie algebras of Galois groups arising from Hodge-Tate modules, Ann. of Math. 97 (1973), 160-170. Zbl 0258.12009 MR 0314853

[28] J.-P. Serre, Abelian $\ell$-adic representations and elliptic curves, W. A. Benjamin, New York, 1968. Zbl 0186.25701 MR 0263823

[29] Facteurs locaux des fonctions zêta des variétés algébriques (définitions et conjectures), in Séminaire Delange-Pisot-Poitou, 1969/70, exp. 19; also in Collected papers, Vol. II, Springer, Berlin, 1986, 581-592. Zbl 0214.48403

[30] - Propriétés galoisiennes des points d'ordre fini des courbes elliptiques, Invent. Math. 15 (1972), 259-331. Zbl 0235.14012 MR 0387283

[31] Congruences et formes modulaires (d'après H. P. F. Swinnerton-Dyer), in Séminaire Bourbaki, 1971/72, exp. 416, Lecture Notes in Math. 317, Springer, Berlin, 1973, 319-338. Zbl 0276.14013 MR 0466020

[32] _ Représentations linéaires des groupes finis, 5ème éd., Hermann, Paris, 1998. Zbl 0926.20003

[33] J.-P. Serre and J. Tate, Good reduction of abelian varieties, Ann. of Math. 88 (1968), 492-517. Zbl 0172.46101 MR 0236190

[34] J. Suh, Symmetry and parity in Frobenius action on cohomology, Compos. Math. 148 (2012), 295-303. Zbl 1258.14023 MR 2881317

[35] T. Tsuji, $p$-adic étale cohomology and crystalline cohomology in the semi-stable reduction case, Invent. Math. 137 (1999), 233-411. Zbl 0945.14008 MR 1705837 